Article

\title{
Anomalous Heat Transport in Nanolaminate Metal/Oxide Multilayer Coatings: Plasmon and Phonon Excitations
}

\author{
Anatoly Kovalev ${ }^{1}$, Dmitry Wainstein ${ }^{1, *} \mathbb{0}$, Vladimir Vakhrushev ${ }^{1}$, Raul Gago ${ }^{2} \mathbb{C}$ \\ and Jose Luis Endrino 3,4 \\ 1 Surface Phenomena Researches Group, I.P. Bardin Central Research Institute for Ferrous Metallurgy, \\ 105005 Moscow, Russia; a_kovalev@sprg.ru (A.K.); gareq1211@gmail.com (V.V.) \\ 2 Instituto de Ciencia de Materiales de Madrid, Consejo Superior de Investigaciones Científicas, \\ E-28049 Madrid, Spain; rgago@icmm.csic.es \\ 3 Basque Center for Materials, Applications \& Nanostructures, UPV/EHU Science Park, Barrio Sarriena s/n, \\ 48940 Leioa, Spain; jose.endrino@bcmaterials.net \\ 4 IKERBASQUE, Basque Foundation for Science, Maria Diaz de Haro 3, 48013 Bilbao, Spain \\ * Correspondence: d_wainstein@sprg.ru; Tel./Fax: +7-495-777-94-10
}

Received: 27 January 2020; Accepted: 10 March 2020; Published: 11 March 2020

\begin{abstract}
In this work, the anomalous reduction in the thermal conduction observed for nanolaminate metal-dielectric multilayers has been extended to the case of oxides. For this purpose, $\mathrm{Ag} / \mathrm{Al}_{2} \mathrm{O}_{3}$ coatings were produced with different layer thicknesses (from 1 to $5 \mathrm{~nm}$ for Ag and 8 to $40 \mathrm{~nm}$ for $\mathrm{Al}_{2} \mathrm{O}_{3}$ ) and numbers of stacks. It was found that the thermal conduction is significantly lower in such metal-oxide nanolaminates compared to the bulk oxide. Such anomalous behaviour is explained by the influence of plasmon and phonon propagation confinement in nanolayers and at the interfaces. To this end, the characteristics of the different types of acoustic and optical phonon waves propagating in the multilayer coating have been studied. In particular, the electronic structures of the different layers and their influences on the plasmon resonance are investigated as a function of the multilayer design. The plasmon-polariton mechanism of energy transfer through oxide-metal and metal-oxide interfaces is discussed.
\end{abstract}

Keywords: multilayer $\mathrm{Al}_{2} \mathrm{O}_{3} / \mathrm{Ag}$ heterostructures; physical vapour deposition (PVD); thermal conductivity; interface; size effect; plasmon; phonon propagation; confinement; high resolution electron energy loss spectroscopy (HREELS)

\section{Introduction}

Presently, it is well known that miniaturization to the nanoscale in multilayer coatings (planar metamaterials) allows one to change, sharply, many of their physical properties, including electronic and optical ones. Such responses result from strong transformations in the electronic structures of the nanoscale materials, which, for example, can be reflected in shift of the binding energies of core-levels as measured by X-ray photoelectron spectroscopy (XPS) [1] or the frequency shifts of Raman modes in the so called "optical mode softening" and "acoustic mode hardening" [2]. Special attention should be paid to metal-insulator-metal (MIM) multilayer coatings that can be considered as the new class of planar metamaterials [3]. Electronic or atomic oscillations in MIM composites can transfer or transduce external electromagnetic radiation overrunning sub-diffraction limits [4]. Spatial localization of these wave processes, electron-phonon coupling and scattering directly influence the absorption and emission of electromagnetic waves in lot of optoelectronic devices and device transport dynamics [5]. Predicted specific optical or electromagnetic properties of such metamaterials are based on anomalies of 
phonons and plasmon propagation in nanostructures [6] that also control the heat conductivities of the materials [7-11]. Therefore, there are good prospects to get multifunctional coatings with favourable combination of strength, optical, and heat protecting properties. Many theoretical studies address them through use in thermoelectric systems [12,13], optics and radiophysics [14], or optoelectronics [3,15,16]. The combination of specific optical and heat protecting properties is required for microlasers, whose power is limited by the heat damage of mirrors [17]. Another potential application of such MIM coatings could be protection of solar cells, including ones operating at high IR intensity [18], but the understanding of heat transfer mechanisms in such systems is still unclear.

The physical nature of heat transmission and scattering in multilayer systems has been well described by the models presented in $[8,10,18,19]$ but the problem consists of inadequate extrapolation of these methods to nanomaterials taking into account the complicated evolution of their properties in the size range from hundreds down to a few nanometres. It was shown that surface states and quantum phenomena control the electronic structures of nanomaterials differently. The first ones prevail for relatively large dimensions, while the second ones are strongly variable with the nanomaterials' electronic structures below several tens of nanometres. This was clearly demonstrated from size shifts of XPS lines in nanomaterials with various compositions and morphologies [20-24]. It is very hard to imagine the generalized response of surface states and quantum phenomena on the actuation of electronic and lattice subsystems by external electromagnetic waves, and this is even more complicated for MIM planar metamaterials. At the same time, there are data on the strong influence of the quantum effects on plasmon and phonon oscillations' propagation in multilayer MIM coatings [25-27]. Hence, the question of interconnections between the heat conductivities of nanomaterials and their surface and quantum states is still not solved. Previously, we addressed said question in multilayer coatings with metal/nitride layers [25-27]. In this case, Ag was used as metal and a wurzite TiAlN layer [27] was used as dielectric media. In the present case, we extend our studies to oxide layers by using $\mathrm{Al}_{2} \mathrm{O}_{3}$ as the dielectric (insulating) layer. In particular, we address size effects (i.e., layer thickness) in the propagation of plasmons and phonons as heat carriers controlling the mechanisms of heat conductivity.

\section{Materials and Methods}

The set of multilayer $\mathrm{Al}_{2} \mathrm{O}_{3} / \mathrm{Ag}$ coatings was fabricated by DC-magnetron sputtering. The thicknesses of individual layers were varied from a few to several hundreds of nanometres. All the samples were covered with a protective top-most $200 \mathrm{~nm} \mathrm{Al}{ }_{2} \mathrm{O}_{3}$ layer to avoid silver oxidation before performing the electron spectroscopy experiments. Table 1 contains parameters of coatings that were used to investigate the difference between multilayer and monolithic coatings.

Table 1. Multilayer coatings composition parameters.

\begin{tabular}{ccccc}
\hline Sample Code & $\begin{array}{c}\mathbf{A l}_{\mathbf{2}} \mathbf{O}_{\mathbf{3}} \text { Single Layer } \\
\text { Thickness, } \mathbf{n m}\end{array}$ & $\begin{array}{c}\text { Ag Single Layer } \\
\text { Thickness, } \mathbf{n m}\end{array}$ & $\begin{array}{c}\text { Number of } \\
\mathbf{A l}_{\mathbf{2}} \mathbf{O}_{\mathbf{3}} \mathbf{\text { Ag }} \text { Bilayers }\end{array}$ & $\begin{array}{c}\text { Total Thickness, } \\
\mathbf{n m}\end{array}$ \\
\hline $5 / 40$ & 40 & 5 & 4 & 180 \\
$2 / 40$ & 40 & 2 & 4 & 168 \\
$4 / 20$ & 20 & 4 & 7 & 168 \\
$1 / 8$ & 8 & 1 & 7 & 63 \\
$2 / 24$ & 24 & 2 & 2 & 52 \\
$5 / 24$ & 24 & 5 & 2 & 58 \\
200 & 200 & 0 & 1 & 200 \\
\hline
\end{tabular}

The layer structure of the coatings was verified by Rutherford backscattering spectrometry (RBS). The measurements were performed with the 5 MV Cockroft-Walton tandetron at the Centro de Microanálisis de Materiales (CMAM) in Madrid (Spain) using a $2 \mathrm{MeV} \mathrm{He}^{+}$probing beam, and the backscattered ions were detected with a silicon detector at a scattering angle of $170^{\circ}$. The experimental RBS spectra were compared with simulated ones obtained from the SIMNRA code [28] assuming the corresponding sample layer structure. 
The electron spectroscopy investigations were performed using the multifunctional electron spectrometer ESCALAB MK2 (VG, East Grinstead, UK) equipped by a monochromatic Al K $\alpha$ X-ray source $(h v=1486.6 \mathrm{eV})$, a scanning electron gun LEG200, and an EMU-50 source of monochromatic electrons $\left(E_{0}=1-100 \mathrm{eV}\right)$. Carbon 1's photoelectron peak with binding energy $285.0 \mathrm{eV}$ was used as an internal reference to determine precisely the energy positions of other spectral lines. Prior to the analysis, the samples were sputter cleaned using $\mathrm{Ar}^{+}$ions in the preparation chamber of the spectrometer at a pressure of $10^{-5} \mathrm{~Pa}$, an accelerating voltage of $5 \mathrm{kV}$, and a current of $20 \mu \mathrm{A}$. The precise control over the lateral $\mathrm{Ar}^{+}$ion etching profile attained with the AG- 6 ion gun was used to fabricate $0.25^{\circ}$ angle laps. Additionally, ion etching with simultaneous sample rotation was used for homogeneous thinning the $\mathrm{Al}_{2} \mathrm{O}_{3}$ blanket from $200 \mathrm{~nm}$ to $10 \mathrm{~nm}$, its subsequent removal, and for uniform thinning of $\mathrm{Ag}$ layers. The parameters of spectra acquisition were selected to provide high energy spectral resolution with a full width at half maximum (FWHM) about $20 \mathrm{meV}$ for high resolution electron energy loss spectroscopy (HREELS) investigations. Auger electron microscopy (AEM) and electron microscopy of characteristic losses were applied for imaging of the elemental mapping and plasmon intensity distribution through the small-angle cross-section of the multilayer coatings in both individual layers and at interfaces with primary electron energies, $E_{0}$, of 1371.4 and $1401.5 \mathrm{eV}$. The resolution of images was $256 \times 256$ pixels at $10,000 \times$ magnification. The topographic contrast was suppressed by image acquisition software.

Thermal conductivity of the as-deposited coatings was measured by a pulsed photothermal reflectance (PPR) technique described in detail in [29].

\section{Results and Discussion}

\subsection{Fundamentals of Thermal Transport in Metals and Dielectrics}

The thermal conductivity of the $\mathrm{Al}_{2} \mathrm{O}_{3} / \mathrm{Ag}$ multilayer coating can be described by a two-phase system $\left(1=\mathrm{Ag}, 2=\mathrm{Al}_{2} \mathrm{O}_{3}\right)$ consisting of two repetitive nanolayers with thicknesses $L_{1}$ and $L_{2}$, thermal conductivities $K_{1}$ and $K_{2}$, and thermal resistance of a boundary between layers $R_{\mathrm{b}}$. Therefore, the generalized thermal conductivity coefficient $K_{\mathrm{ml}}$ of the multilayer coating with $\mathrm{N}$ bilayers can be described phenomenologically as [30]:

$$
K_{\mathrm{ml}}=\frac{N\left(L_{1}+L_{2}\right)}{R_{\mathrm{ml}}}=\frac{N\left(L_{1}+L_{2}\right)}{\left(\frac{L_{1}}{K_{1}}+\frac{L_{2}}{K_{2}}\right)+(N-1) R_{\mathrm{b}}}
$$

According to Equation (1), the thermal conductivity decreases as the layers become thinner due to growing influence of interfaces. One shall take into account that thermal conductivities of nanolayers also decrease with thinning comparing to "bulk" reference values. Moreover, the thermal conductivity of metals is the sum of lattice (l) and electron (e) contributions:

$$
K_{1}=K_{\mathrm{e}}+K_{1}
$$

However, these contributions are interrelated and also depend on the solid temperature. The phonon contribution in thermal conductivity is 10 times lower than the electronic one for Ag thin films [31].

The ability of electrons to transport heat, for instance, is hindered if they keep bumping into phonons (the vibrating lattice). For this reason, metals have a higher electrical resistivity at higher temperatures. In fact, the heat transport in a pure metal is mostly due to electron-phonon interactions that disrupt the momentum of conducting electrons. Non-metals tend to have a lower thermal conductivity, unless their phonons can carry heat efficiently, and, in essence, have no free electrons. 
In dielectrics with a low density of free electrons, the electronic conduction heat transfer mechanism could be discarded. The phonons part of thermal conductivity consist of three components:

$$
K_{1}=K_{\mathrm{L}}+K_{\mathrm{t} 0}+K_{\mathrm{tu}}
$$

where $K_{L}$ is the longitudinal phonon contribution (acoustic phonons), whereas $K_{\mathrm{t}}$ and $K_{\mathrm{tu}}$ are the contributions of low and high frequency transverse phonons, respectively. Acoustic oscillations play a major role in heat transfer by phonons. An important attribute of heat transfer is the phonon wavelength, because phonon propagation in the media and through the interphase boundaries is a wave process. For example, when the phonon mean free path becomes comparable to the layer thickness in the multilayer coating, the heat propagation perpendicular to interfaces is weakened due to nanoscale effects, as assessed in the thermal transportation of various dielectric materials [32]. Here, the authors introduce the concept of critical acoustic thickness of a film blocking heat transfer by phonons.

Electronic heat transport is the prevailing mechanism in metals, and it is controlled by electronic structure. According to our previous studies [1,20-24], it is drastically rearranged on the nanoscale. These theoretical views are the basis of the present research.

\subsection{Structure of the Multilayer Coatings Revealed by Rutherford Back-Scattering}

Figure 1 shows the experimental (dots) and simulated (solid lines) RBS spectra of representative $\mathrm{Al}_{2} \mathrm{O}_{3} / \mathrm{Ag}$ multilayer coatings. The upper energy limits corresponding to the detected signals from different elements are labelled for identification. The maximum energy of backscattered projectiles from a certain element present at the surface is given by the kinematic factor of the binary collision. For heavier elements than the projectile, this energy increases with the atomic number of the scattering centre, and therefore, backscattered particles are detected at higher energy. Events (counts) from the same element detected at a lower energy correspond to collisions taking place deeper from the surface, since part of the projectile energy is lost on the way in and out through the sample. In this way, RBS provides information about the elemental profile. Furthermore, the RBS yield for each element is proportional to the convolution of its areal density and corresponding Rutherford cross-section (which is proportional to the square of the atomic number), providing information about its concentration at certain depth.

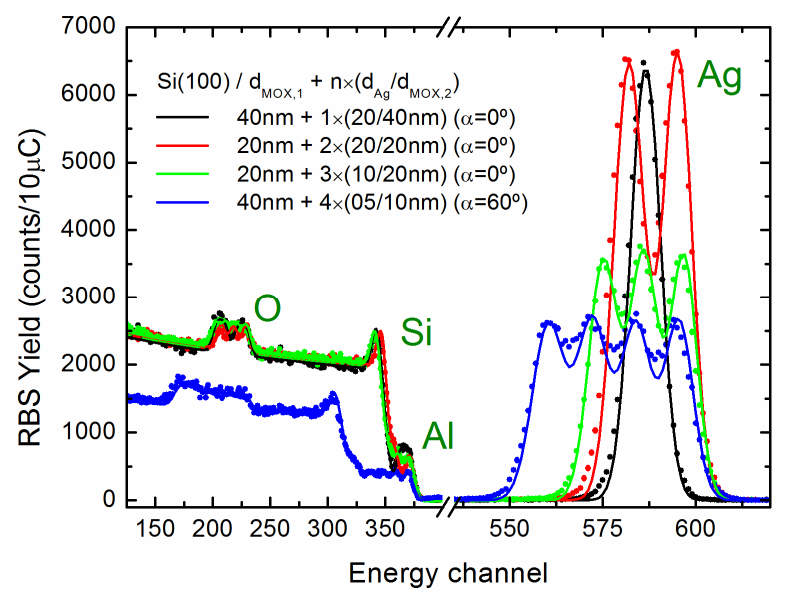

Figure 1. RBS experimental (dots) and simulated (solid lines) spectra from representative multilayer coatings (see label for each structure). The multilayer structure can be extracted from the modulation of the Ag signal. In order to resolve the thinnest Ag layers $(5 \mathrm{~nm})$, the incidence angle $(\alpha)$ of the probing $\mathrm{He}^{+}$beam with respect to the surface normal was increased to $60^{\circ}$ (larger effective layer thickness). The agreement between the simulated and experimental data indicates the good quality of the interfaces. 
In Figure 1, the Ag signal appears well separated on the high-energy side of the spectra due to its relatively high mass with respect to the other film-containing elements and the substrate. In addition, its high atomic number $\mathrm{Z}$ provides a relatively high RBS yield. In all the cases, the coatings present a top-most $\mathrm{Al}_{2} \mathrm{O}_{3}$ layer, and therefore, the energy maximum of the $\mathrm{Ag}$ signal shifts depending on the thickness of said layer due to the secondary scattering of reflected ions on electron shells of atoms located at various depths in the coating. The modulation of the Ag signal is a direct fingerprint of the multilayer structure where the number of maxima indicates the number of Ag layers (n). In order to resolve the thinnest $\mathrm{Ag}$ layers $(5 \mathrm{~nm})$, the incidence angle $(\alpha)$ of the probing $\mathrm{He}^{+}$beam with respect to the surface normal was increased to $60^{\circ}$ (larger effective layer thickness). Structure parameters were refined by comparison of experimental and simulated by SIMNRA code [29] data. The agreement between the simulated and experimental data supports the stoichiometry of the different layers and indicates the good quality of the interfaces (within the RBS resolution of $\approx 5 \mathrm{~nm}$ ).

\subsection{Thermal Conductivity of Multilayer Coatings and Interfaces}

To estimate the role of metal-dielectric boundaries in our metal-oxide composites, we evaluated their thermal conductivity $K_{\mathrm{b}}$ in the system based on experimental measurements of thermal conductivities together with literature data on heat conductivities of $\mathrm{Al}_{2} \mathrm{O}_{3}$ and $\mathrm{Ag}$ nanolayers of different thickness using Equation (4) derived from Equation (1):

$$
K_{\mathrm{b}}=\frac{1}{R_{\mathrm{b}}}=\frac{N-1}{\frac{N\left(L_{1}+L_{2}\right)}{K_{\mathrm{ml}}}-\left(\frac{L_{1}}{K_{1}}+\frac{L_{2}}{K_{2}}\right)}
$$

Figure 2 presents heat conductivity coefficients of the multilayer coatings $\left(K_{\mathrm{ml}}\right)$ and the relative contribution of interfaces $\left(K_{\mathrm{b}} / K_{\mathrm{ml}}\right)$ as function of the bilayer thickness. These values have been extracted from the measured thermal conductivities of the multilayered samples presented in Table 1 together with the reported thermal conductivity data for bulk and nanolayer alumina [31,33-36] and Ag [36-38] by interpolating and extrapolating them to the layer thicknesses of our samples. The experimental data obtained from our multilayer coatings are plotted in the region below $50 \mathrm{~nm}$. The results for bilayers thicknesses above from $50 \mathrm{~nm}$ were calculated on the basis of literature data using Equation (1).

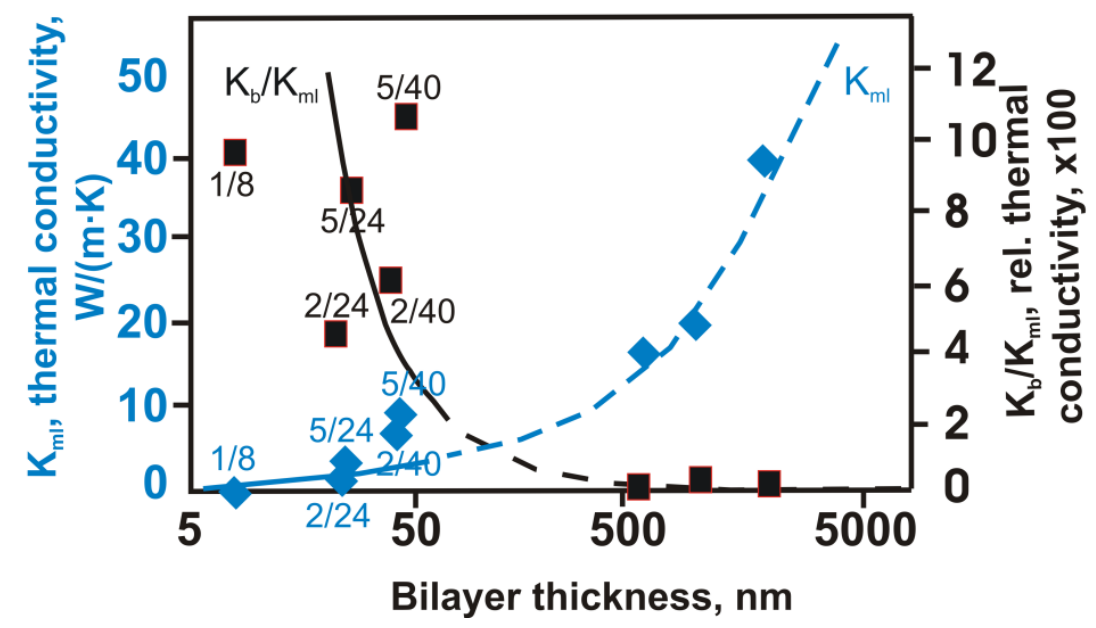

Figure 2. Influence of the $\mathrm{Ag} / \mathrm{Al}_{2} \mathrm{O}_{3}$ bilayer thickness on the thermal conductivity coefficients of the multilayer coatings $\left(K_{\mathrm{ml}}\right)$, and the relative thermal conductivity of interfaces $\left(K_{\mathrm{b}} / K_{\mathrm{ml}}\right)$. The values below $50 \mathrm{~nm}$ (solid line) correspond to the experimental data from this work; corresponding points are marked with sample codes. Values above $50 \mathrm{~nm}$ (dashed line) are results of calculations by using Equation (1) and literature data. 
Thermal conductivity increases to $\approx 10 \mathrm{~W} / \mathrm{m} \cdot \mathrm{K}$ when bilayer thickness increases from $\approx 25 \mathrm{~nm}$ to around $45 \mathrm{~nm}$ for the Ag layer thickness of 2 and $5 \mathrm{~nm}$; that is approximately three times lower than thermal conductivity of alumina $28.84 \mathrm{~W} /(\mathrm{m} \cdot \mathrm{K})$ found in the sample 200. Therefore, an addition of the component with high thermal conductivity $\left(K_{\mathrm{Ag}}=430 \mathrm{~W} /(\mathrm{m} \cdot \mathrm{K})\right.$ in the "bulk" state) decreases, paradoxically, the thermal conductivity of multilayer system. As the theoretical calculation shows, the thick coating $(4500 \mathrm{~nm} / 4500 \mathrm{~nm})$ has a high thermal conductivity of about $202.0 \mathrm{~W} /(\mathrm{m} \cdot \mathrm{K})$.

The average $K_{\mathrm{b}}$ value was $11.0 \mathrm{~W} / \mathrm{K}$ with variation from 0.27 to $25.9 \mathrm{~W} / \mathrm{K}$. Such variation may occur due to unequal conditions of measurements and calculations in the above-mentioned sources, but the curve $K_{\mathrm{b}} / K_{\mathrm{ml}}$ on Figure 2 clearly illustrates the tendency in the growing role of interfaces.

One can see that interfaces make a significant input to heat transfer only in nanolaminate metal-dielectric structures with bilayers thinner than $150 \mathrm{~nm}$. This trend corroborates the hypothesis that the role of interfaces becomes more relevant as materials downsize toward the nanoscale. Heat dissipation at the interface in thick multilayer coatings is considerably lesser in comparison with the thermal conductivity of the volume.

Generally, the thermal conductivity of nanosystems strongly depends on its geometry [39] or roughness regarding interfaces, but in our case we have enough perfectly smooth boundaries between layers (see Figure 1and Figure 10) to assume the same $K_{\mathrm{b}}$ values for all samples. Moreover, it will be shown below that metal-oxide and oxide-metal boundaries are not symmetric relative to heat transfer direction.

\subsection{Electronic Structure and Features of Plasmon and Phonon Propagation in $\mathrm{Al}_{2} \mathrm{O}_{3} / \mathrm{Ag}$ Multilayer Coatings}

Studies of $\mathrm{Ag} / \mathrm{Al}_{2} \mathrm{O}_{3}$ nanolaminate coatings' electronic structures and features of the phonon and plasmon oscillations' propagations enabled explaining their anomalously low thermal conductivity.

Figure 3a shows the XPS spectrum of the $\mathrm{Al}_{2} \mathrm{O}_{3}$ layer valence band with individual components resolved after deconvolution and fitting. This oxide has a cubic nanocrystalline structure where oxygen states are dominant. The upper valence levels correspond to the $\mathrm{O} 2 \mathrm{p}$ non-bonding states, and the lower part is the $\mathrm{O} 2 \mathrm{p}$ bonding states mixed with the $\mathrm{Al} 3 \mathrm{p}$ electrons. The valence band (VB) component with maximal binding energy corresponds to $\mathrm{Al} 3 \mathrm{~s}$ orbitals. These data are in good agreement with theoretically calculated $\mathrm{Al}_{2} \mathrm{O}_{3}$ band structure [40,41]. High localization of $\mathrm{O} 2 \mathrm{p}-\mathrm{Al} 3 \mathrm{p}$ bonds and a small electron concentration near the Fermi level are crucial factors determining thermal properties: decreasing of electrons concentration near Fermi level limits electronic transport into conducting band and decreases electronic input in heat conductivity.

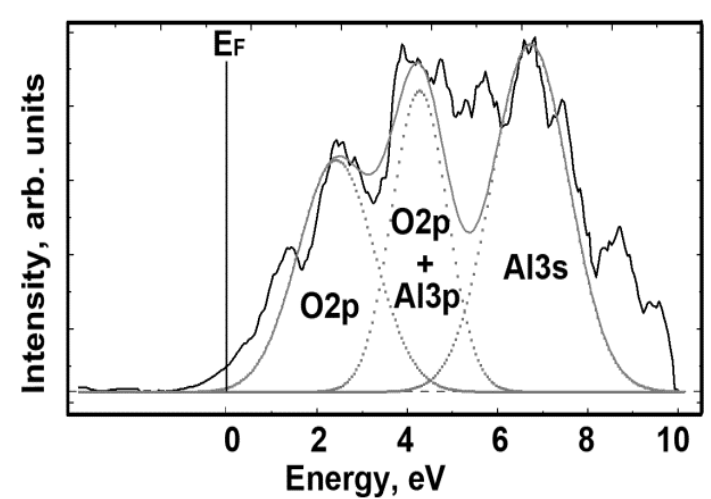

(a)

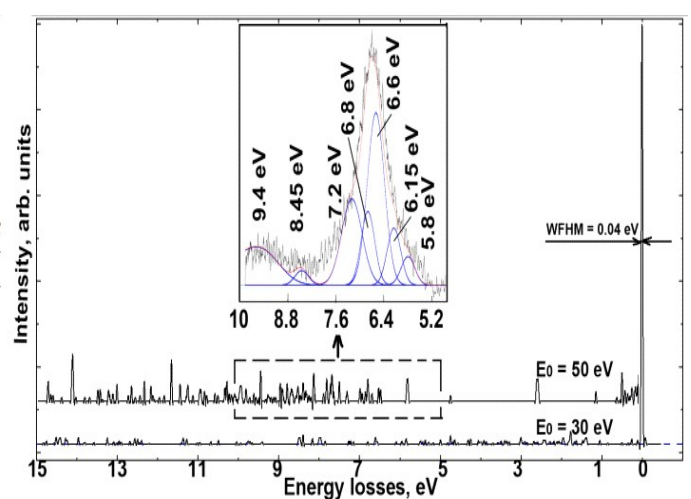

(b)

Figure 3. Fine structure of $170 \mathrm{~nm} \mathrm{Al} \mathrm{O}_{3}$ layer valence band obtained by XPS (a) and interband transitions spectra, excited by primary electron beams with energies of 30 and $50 \mathrm{eV}$ (b). The full width at half maximum (FWHM) of the back scattered electron peak is $40 \mu \mathrm{eV}$. The dotted lines are displaying fitted components of valence electrons and interband transitions. 
HREEL spectra from $170 \mathrm{~nm}$ layer $\mathrm{Al}_{2} \mathrm{O}_{3}$ at primary electrons energies of 30.0 and $50.0 \mathrm{eV}$ are shown in Figure 3b. We shall remark that noticeable excitation of interband electronic transitions can be detected at primary electron energies over $30 \mathrm{eV}$. O 2p and $\mathrm{Al} 3 p$ electrons form strong covalent bonds localizing far from the Fermi level. This explains the great potential of their excitation for transitions from valence to the conduction band (CB). Interband electronic transitions in the range of $5.0-10.0 \mathrm{eV}$ are shown as insets in Figure $3 \mathrm{~b}$ at the electron source energy of $50.0 \mathrm{eV}$. The fine structure of the energy transitions was fitted by Gaussian peaks. Differences in components consolidated in two groups 5.8-7.2 eV and 8.45-9.4 eV are clearly visible. The interpretation of these transitions has been made on the basis of the theoretical band structure of alumina [38]. A schematic representation of the interband electron transitions across the band gap in $\mathrm{Al}_{2} \mathrm{O}_{3}$ under external excitation is shown in Figure 4 . The wide peak A corresponds to transitions along the $\Gamma$ symmetry direction in the Brillouin Zone (reciprocal lattice). The B peaks appear as a result of the transitions of O2p valence electrons in the conduction band in the directions $\mathrm{X}$ and $\mathrm{M}$.

The HREELS and XPS data enable estimating the influence of nanostructuring on the width of the band gap. For the case of $\mathrm{Al}_{2} \mathrm{O}_{3} 170 \mathrm{~nm}$ layer, the width of band gap which separates the valence and conduction bands corresponds to $5.8-7.2 \mathrm{eV}$ at $\Gamma$ symmetry point. The theoretical value for the $\Gamma$ direction of $\mathrm{Al}_{2} \mathrm{O}_{3}$ band gap is $7.2 \mathrm{eV}$, as found by Mousavi at al. [42]. According to [43], the minimal width of the band gap in $\Gamma$ symmetry point is $4.2 \mathrm{eV}$. Our experimental value of the band gap is very close to the theoretical one. Intensities of HREELS components in the A band correspond to the calculated density of states in the CB.

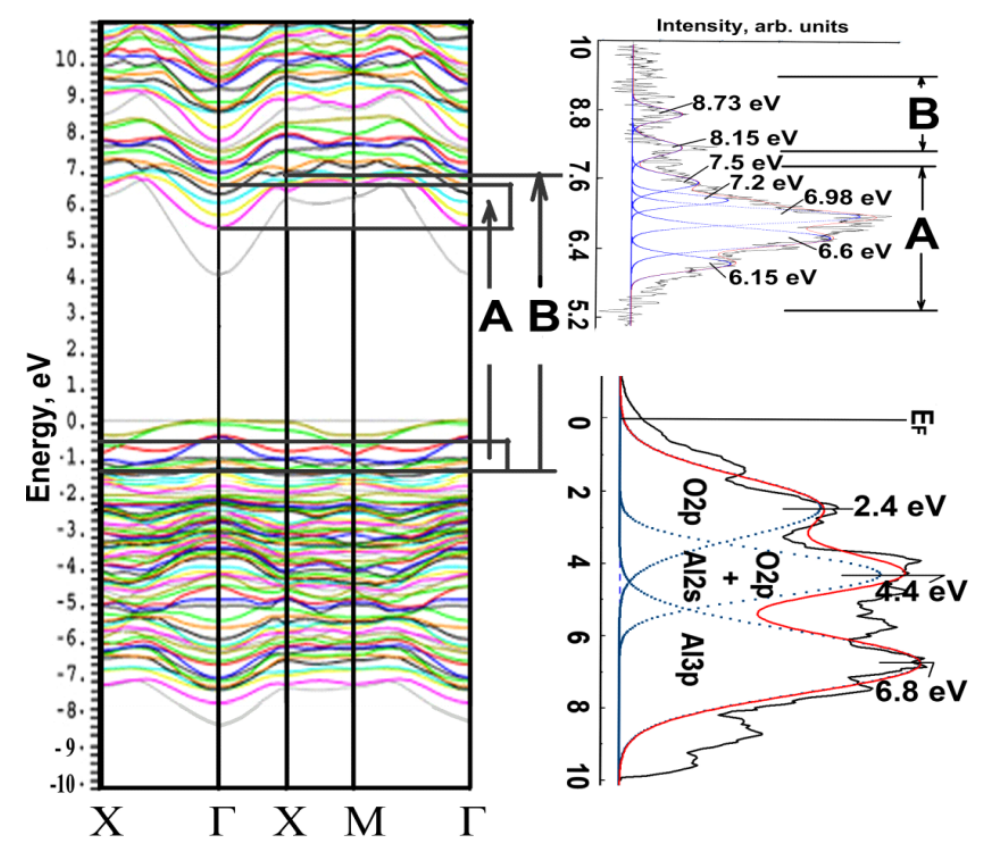

(a)

Figure 4. Interpretation of HREELS fine structure. Calculated density of states (DOS) of $\mathrm{Al}_{2} \mathrm{O}_{3}$ (a) based on data from [32]; energy loss spectrum (b) and valence band (c) $20 \mathrm{~nm}$ layer of $\mathrm{Al}_{2} \mathrm{O}_{3}$.

Figure 5 demonstrates that energies of interband transitions are decreased in the $\mathrm{X}$ and $\mathrm{M}$ directions and increased along the direction $\Gamma$ while $\mathrm{Al}_{2} \mathrm{O}_{3}$ layers are thinned from $170 \mathrm{~nm}$ to $10 \mathrm{~nm}$. This shift is small but detectable. Therefore, the band gap is aligned in alumina layers thinner than approximately $50 \mathrm{~nm}$. The FWHM of HREELS peak characterizing the interband transition in $\Gamma$ direction is $0.80 \mathrm{eV}$. This relatively large width of the "A" HREELS line indicates that there is good mobility for electrons if they only could jump over wide band gap. 


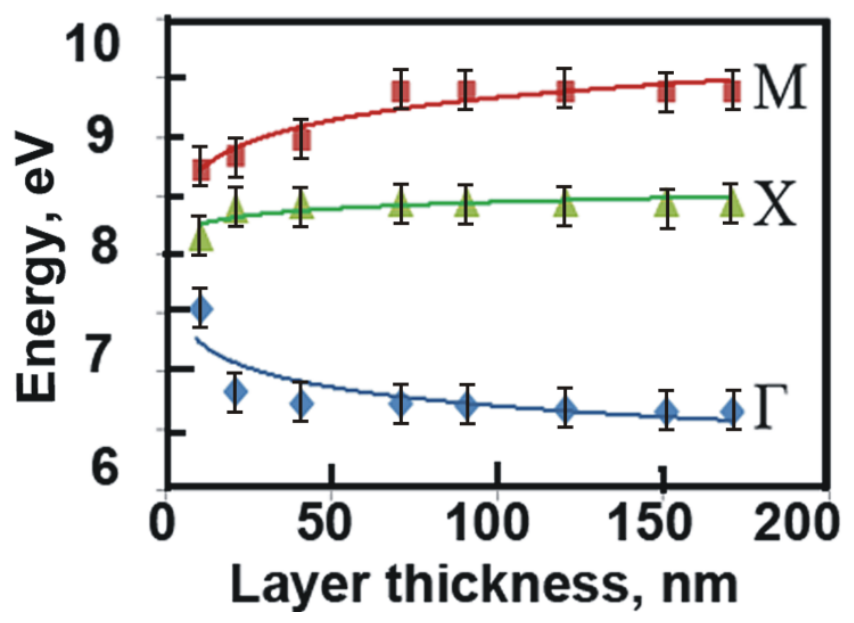

Figure 5. Energy positions of HREELS intensive peaks characterizing interband transitions in $\Gamma, X$, and $\mathrm{M}$ symmetry directions in the Brillouin zone of $\mathrm{Al}_{2} \mathrm{O}_{3}$ layers.

The electronic structure of silver was also changed with nanostructuring. Binding energies of inner $\mathrm{Ag} 3 \mathrm{~d}$ levels are increased with thinning $\mathrm{Ag}$ layers in the $\mathrm{Ag} / \mathrm{Al}_{2} \mathrm{O}_{3}$ multilayer coating (Figure 6). A similar appearance was observed earlier in nanocrystals and layers of various materials with refinements of their characteristic sizes at the nanoscale [20-22,26]. The decreasing of Ag thickness induces notable changes in valence band of $\mathrm{Ag}$ [26]. Fermi level is shifted deeper and DOS near $\mathrm{E}_{\mathrm{F}}$ is decreased. In a thin film state, a metal acquires the properties of the dielectric. One can assume that transformations in the electronic structure of $\mathrm{Al}_{2} \mathrm{O}_{3}$ and $\mathrm{Ag}$ are naturally affected by plasmons and phonons propagation in the volume and near interfaces, contributing to the general mechanism of heat transfer.

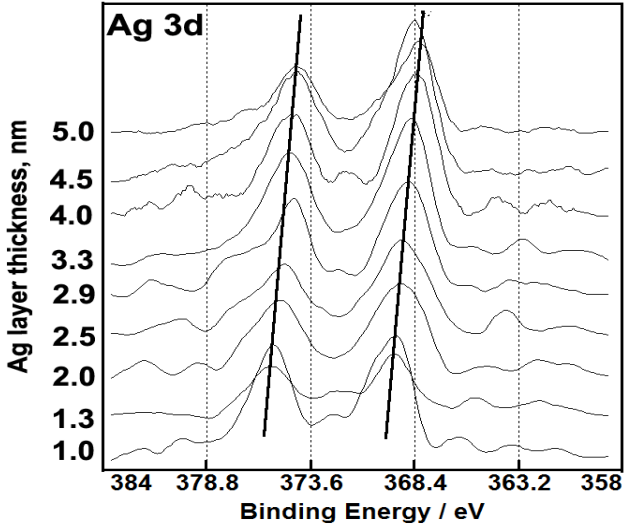

(a)

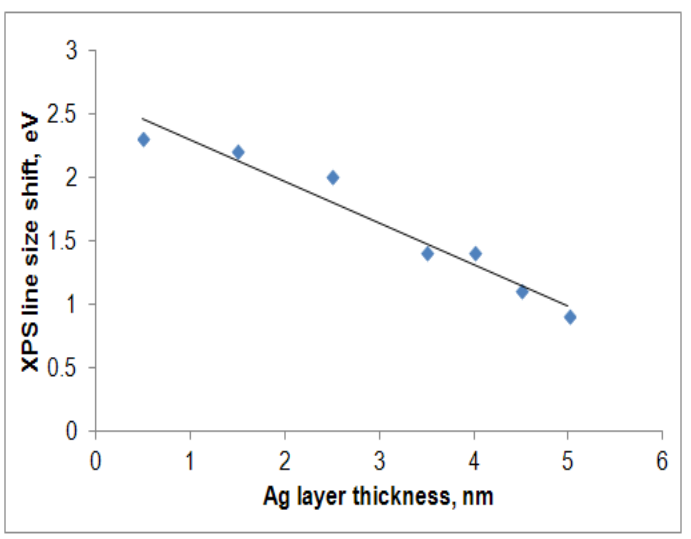

(b)

Figure 6. Size shift of $\mathrm{Ag} 3 \mathrm{~d}$ binding energies relative to the Ag reference: the set of photoelectron lines from Ag layers with different thicknesses (a); size shifts as functions of Ag layer thickness (b).

The original and deconvoluted vibrational (phonon) spectra from 170, 120, and $80 \mathrm{~nm} \mathrm{Al}_{2} \mathrm{O}_{3}$ layers obtained by ion beam thinning of the $200 \mathrm{~nm} \mathrm{Al}{ }_{2} \mathrm{O}_{3}$ protective layer above the $\mathrm{Ag} / \mathrm{Al}_{2} \mathrm{O}_{3}$ multilayers are shown on Figure 7. Alumina has a rhombohedral elementary cell containing 10 atoms, and therefore, 30 phonon branches (modes) could be excited [44]. The HREELS can resolve only a small part of the strongest vibrations. The intensity of phonon spectra is decreased with thinning of the alumina layer. One can see on Figure $7 \mathrm{~b}$ that experimental HREELS spectra have a complex structure, containing acoustic (TA) and optical (TO) components in two ( $\Gamma$ and $X)$ symmetry directions in the Brillouin zone. Deconvolution and subtracting one spectrum $(170 \mathrm{~nm})$ from the other one $(80 \mathrm{~nm})$ amplifies some of 
the finest changes of phonon propagation with the decreasing of $\mathrm{Al}_{2} \mathrm{O}_{3}$ layer thickness. The resulting spectrum in Figure 7c shows that first acoustic oscillations are slightly reduced in intensity when layer thickness is decreased from $170 \mathrm{~nm}$ to $80 \mathrm{~nm}$. The first and second harmonics of longitudinal (LO) and transverse (TO) optical oscillations of $\Gamma$ and $X$ directions are attenuated significantly in the $80 \mathrm{~nm}$ layer relatively to $170 \mathrm{~nm}$ one. Comparing optical and acoustic vibrations, one can see that the first is attenuated stronger. The higher-order harmonics are attenuated in thin layers. When decreasing the layer thickness to $2.0 \mathrm{~nm}$, the acoustic and optical phonon peaks are shifted to shorter wavelengths. This "blue shift" can be clearly seen at layers thicknesses below $80.0 \mathrm{~nm}$, which corresponds to results reported in [41].

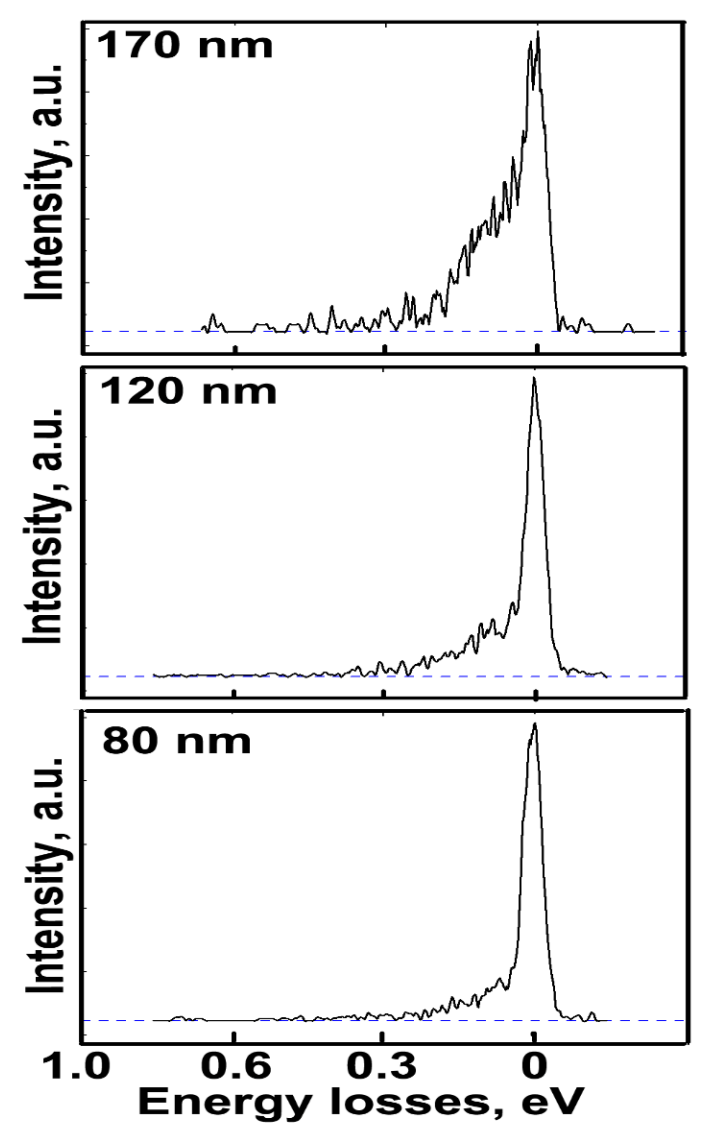

(a)

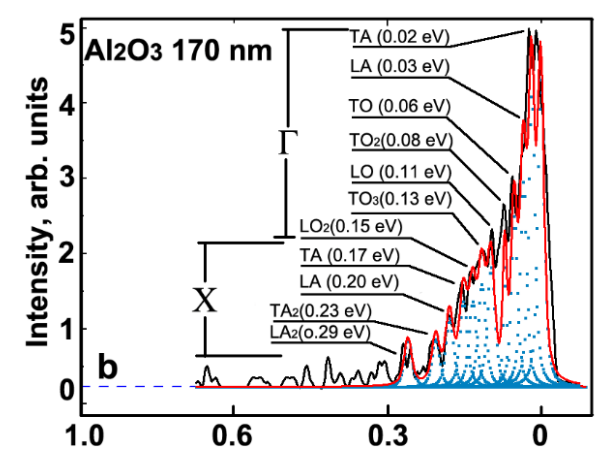

(b)

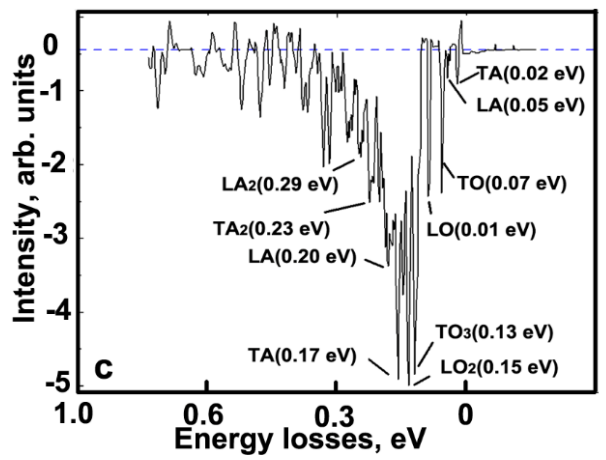

(c)

Figure 7. Comparison of phonon HREEL spectra of 170, 120, and $80 \mathrm{~nm} \mathrm{Al}_{2} \mathrm{O}_{3}$ layers (a); fine structure of phonon spectra in $170 \mathrm{~nm} \mathrm{Al}_{2} \mathrm{O}_{3}$ layer $(\mathbf{b})$; the differentiation of HREELS acquired from $80 \mathrm{~nm}$ and $170 \mathrm{~nm} \mathrm{Al}_{2} \mathrm{O}_{3}$ layers (c). 
This phenomenon was dissimilar for acoustic and optical excitations with different polarizations. Figure 8 depicts changes of anisotropy parameters for acoustic $\left(m_{\mathrm{A}}\right)$ and optical $\left(m_{\mathrm{O}}\right)$ phonon modes calculated through expressions:

$$
m_{\mathrm{A}}=\omega_{\mathrm{LA}} / \omega_{\mathrm{TA}}
$$

and

$$
m_{\mathrm{O}}=\omega_{\mathrm{LO}} / \omega_{\mathrm{TO}}
$$

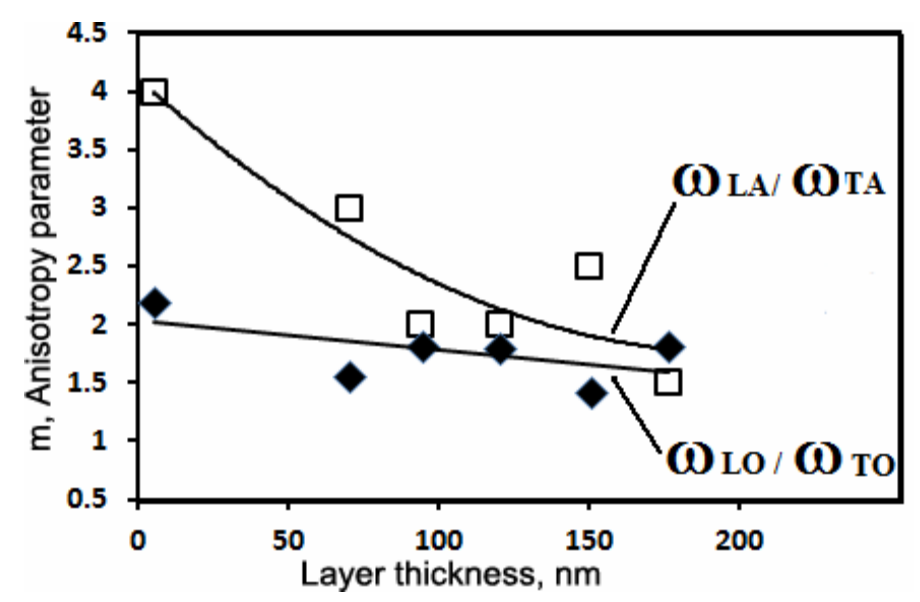

Figure 8. The dependence of phonon propagation anisotropy parameters $\left(m_{\mathrm{A}}, m_{\mathrm{O}}\right)$ on $\mathrm{Al}_{2} \mathrm{O}_{3}$ layer thickness.

The anisotropy of acoustic modes increases noticeably in insulator layers thinner than $80 \mathrm{~nm}$, while frequencies of $\mathrm{LO}$ and TO vibrations have smaller dependencies on layers thickness. The blue shift of transverse acoustic (TA) phonons was more visible than longitudinal (LA) ones at decreased thicknesses of the oxide layer. TA phonon decay is faster in thin layers than in thick ones. That is, the TA phonon coupling is increased mostly in the $8 \mathrm{~nm}$ layer. It is most likely that such obvious and significantly sized effects are connected to the traditional relationship of phonon mean free path (MFP) with the geometric (crystallographic) parameters of the $\mathrm{Al}_{2} \mathrm{O}_{3}$ layer structure. According to [40], the anisotropy factor of acoustic phonons in $\mathrm{Al}_{2} \mathrm{O}_{3}$ single crystal is approximately 1.13. In our case, the parameter " $m$ " is slightly higher, and it increases significantly in thin films due to anisotropic phonon scattering. As this phenomenon is observed at frequencies controlling heat transport, it will undoubtedly have an effect on the anisotropy of alumina thermal conductivity.

Heat dissipation in heterophase materials occurs not only in the volume, but also at interfaces. In the multilayer nanocomposite, the interfacial thermal resistance plays a critical role for the heat transport. When Phase 1 is $\mathrm{Al}_{2} \mathrm{O}_{3}$ and Phase 2 is $\mathrm{Ag}$, the phonon transmittance probability enables characterizing the energy transfer across the interface [45]:

$$
a_{1, j}(\omega)=\frac{v_{2, j} \cdot g_{2, j}(\omega)}{v_{1, j} \cdot g_{1, j}(\omega)+v_{2, j} \cdot g_{2, j}(\omega)}
$$

where $a_{1, j}$ is phonon transmission probability of mode $j$ in phase $1 ; \omega$ is phonon angular frequency; $v_{i, j}$ is phonon velocity of mode $j$ in phase $\mathrm{I}$; and $g_{i, j}(\omega)$ is the phonon density of states of mode $j$ in side $i$. The latter corresponds to the intensity of the $i^{\text {th }}$ phonon peak on the HREEL spectrum.

The phonon velocity was calculated from

$$
v_{\mathrm{i}}=\omega_{\mathrm{i}}\left(\mathrm{GHz} \cdot \mathrm{sec}^{-1}\right) / q_{\mathrm{i}}
$$

where $q_{1}$ is wavevector for the trigonal $\mathrm{A}_{2} \mathrm{O}_{3}$ Brillouin zone $\mathrm{f}$. A vector magnitude of $14.5 \mathrm{rad} \cdot \mathrm{nm}^{-1}$ was calculated to be nearly perpendicular to the XY plane [40]; $q_{2}$ is a lattice vector 
of $\mathrm{Ag}(\Gamma \Gamma \Lambda)=\frac{\sqrt{3} \pi}{a}=13.3 \mathrm{rad} \cdot \mathrm{nm}^{-1}$, where Ag lattice constant $a=0.4085 \mathrm{~nm}$. The experimental phonon frequencies $\left(\omega_{\mathrm{TA}}\right.$, LA for Ag) and intensities of REEL spectral components ( $g_{\mathrm{TA}}$, LA for $\mathrm{Ag}$ ) were previously obtained experimentally in [26]. Thicknesses of silver layers were chosen equal to alumina ones.

Figure 9 demonstrates that phonons' transmittance through the interface depends on layer thickness and direction of energy transfer. These graphs were calculated based on experimental analysis of the phonon resonance in $\mathrm{Ag}$ and $\mathrm{Al}_{2} \mathrm{O}_{3}$ layers to interpret phonon propagation across boundaries in accordance with the model of Equations (5) and (6). Phonon transmittance across the metal-isolator interface is very minor and follows approximately a linear relation for layer thickness from 80.0 to $2.0 \mathrm{~nm}$. While the layers' thickness is over $100 \mathrm{~nm}$, the probability of transboundary transmittance increases nonlinearly. These changes are similar for each type of phonon polarization. In contrast, the probability of boundary energy transmission in the opposite (dielectric $\rightarrow$ metal) direction decreases monotonically with increasing thickness of the layers.

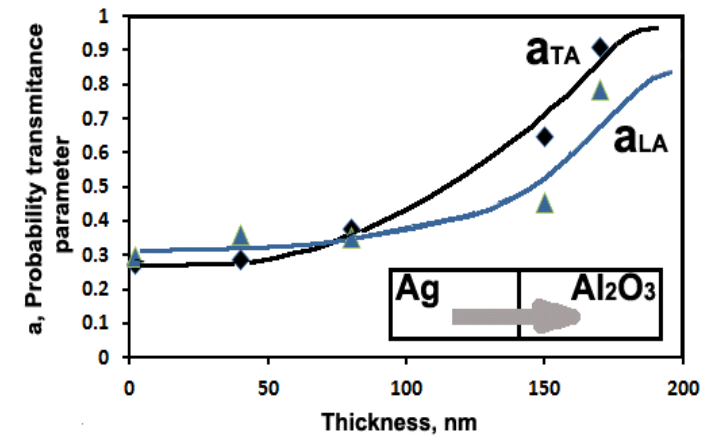

(a)

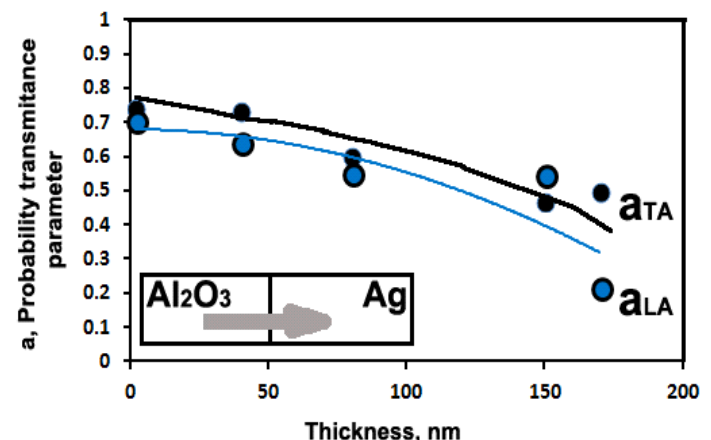

(b)

Figure 9. Probability of phonon energy transmittance across interfaces in $\mathrm{Ag} / \mathrm{Al}_{2} \mathrm{O}_{3}$ layer nanocomposite from Ag to alumina nanolayer (a) and from alumina to silver (b) depending on individual layer thickness and direction of propagation across phase boundary.

In this case, the observed dimensional dependence cannot be explained by the traditional comparison of MFP and layer thickness. Perhaps such a complex and ambiguous nature of energy transfer across the boundary occurs is due to differences in the polarization vector and the phonon energy in each of these cases. The observed size effect of transboundary phonon propagation allows us to explain the enhanced barrier role of interfaces in energy transfer within the multilayer coatings. Probability of phonon heat transfer from silver nanolayers to alumina is extremely low for thin layers. From other side, taking into account that the phonon part of heat transfer in metals is several tens of times lesser than the plasmon mechanism, we can assume that phonon mechanism is substantially weakened in heterophase structures with nano-sized layers. Therefore, we need to study the plasmon contribution to the propagation of heat in the multilayer nanocomposites.

Figure 10a-c display SEM images of angle lap of the $5 / 24 \mathrm{~nm} \mathrm{Ag} / \mathrm{Al}_{2} \mathrm{O}_{3}$ coating acquired with back scattered electrons $\left(E_{0}=1371.4 \mathrm{eV}\right)$, plasmon mapping at energy loss $E_{\mathrm{p}}=4.80 \mathrm{eV}, \mathrm{Ag} \mathrm{MNN}$ Auger imaging (AEM), and line distribution of plasmon intensity along 1-1 line on AEM. On the BEI of Figure 10a, the white contrast is formed in the silver layers; dark areas correspond to alumina. The map of symmetric plasmon excitation with energy of $4.8 \mathrm{eV}$ and the same linear distribution at plasmon energy $4.9 \mathrm{eV}$ are shown in Figure 10b,c. Symmetric or antisymmetric types of plasmon excitation depend on the plasmon energy and thickness of Ag layers. The antisymmetric resonance is observed in layers thicker than $5 \mathrm{~nm}$, while the symmetrical character occurs most likely in a thin metal layer. Figure 10c shows the evolution of plasmon intensity at different energies as the electron probe is line-scanned along the 1-1 direction on the AEI (insert on Figure 10c). The plasmon intensity and its lateral distribution depend on the resonance energy. Plasmons are excited symmetrically 
or antisymmetrically in the silver layer depending on their excitation energy. These experimental observations are in very good agreement with theoretical calculations [46].
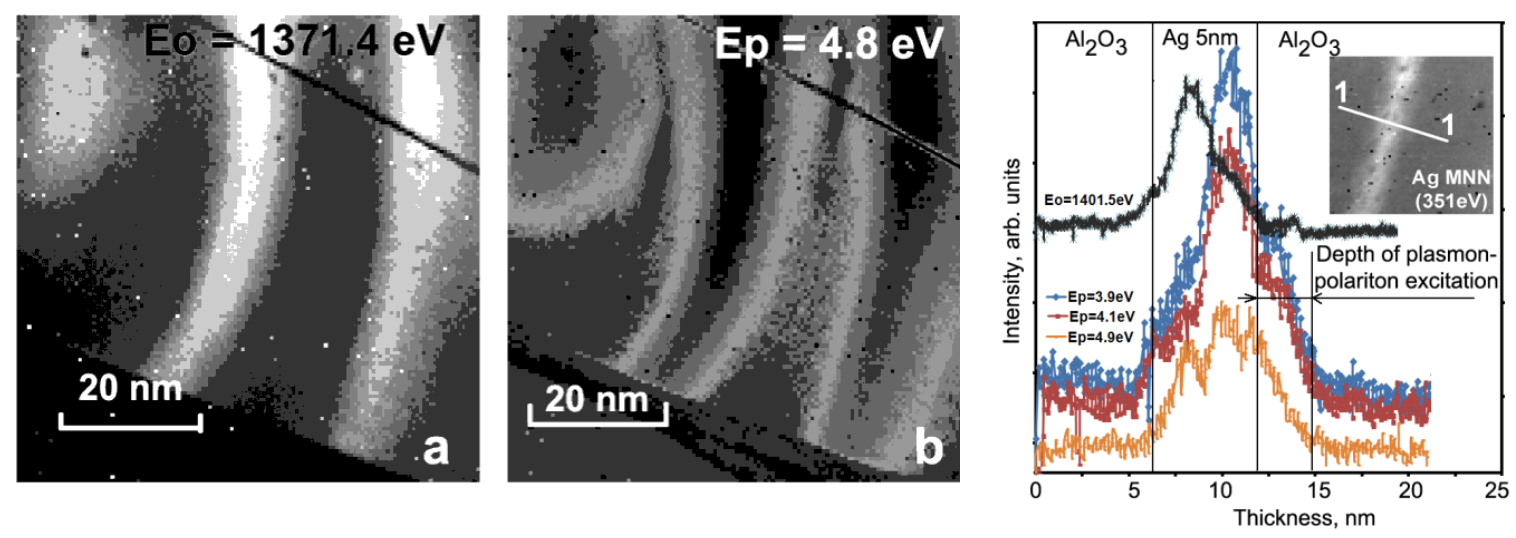

(c)

Figure 10. Structure of $\mathrm{Ag} / \mathrm{Al}_{2} \mathrm{O}_{3}$ 5/24 multilayer coating: (a) back scattered electron image (BEI) of angle lap; (b) map of plasmon excitation at $E_{\mathrm{p}}=4.8 \mathrm{eV}$; (c) linear distribution of plasmon excitations across 1-1 direction on insert of Auger Ag MNN image (AEI). $E_{0}$ is energy of back scattered electrons; $E_{\mathrm{p}}$-energy of plasmon resonance.

Antisymmetric plasmon excitations at 3.9 and $4.1 \mathrm{eV}$ are propagated along phase boundaries. These plasmons are penetrated into the dielectric layer at some depth (h) through the interface due to plasmon-polariton (SPP) interactions. This non-uniform resonance structure is a consequence of confinement of electromagnetic DOS in thin planar nanocomposite. Such a spatial distribution is originated from the confinement of electromagnetic DOS in thin planar nanocomposite. Similar spatially inhomogeneous maps of plasmon excitations were observed earlier in silver nanocrystals with a semi-empirical investigation [47]. The plasmon-polariton penetration into dielectric layers reaches 2.0-5.0 nm, and depends on plasmon energy and excitation intensity. Polariton excitation is the most important stage of electronic heat transfer from metal to dielectric. The depth of plasmon-polariton penetration is the most important characteristic of heat transfer. We assume that the excitation of valence electrons of $\mathrm{Al}_{2} \mathrm{O}_{3}$ with an electromagnetic "plasmon" field existing in the Ag boundary layer is doubtful. Figure $3 a$ demonstrates that $\mathrm{O} 2 \mathrm{p}$ and $\mathrm{Al} 2 \mathrm{~s}$ valence electrons are strongly hybridized. The DOS at the Fermi level is negligible. Interband electron transitions in $\mathrm{Al}_{2} \mathrm{O}_{3}$ can be realized by external energy pumping with sufficient power. On the other hand, we know that the Schottky barrier measured in [48] for an $\mathrm{Ag}$ layer with $35 \mathrm{~nm} \mathrm{Al}{ }_{2} \mathrm{O}_{3}$ film thickness is equal to $1.72 \mathrm{eV}$; it significantly decreases the energy barrier for electron transitions from $\mathrm{Ag} \mathrm{VB}$ to $\mathrm{Al}_{2} \mathrm{O}_{3} \mathrm{CB}$ by external energy pumping (Figure 11).

We assume the mechanism of plasmon-polariton interaction is as follows. We can suppose that Ag conducting electrons are injected into the $\mathrm{CB}$ of the insulator by an external energy source and a boundary electromagnetic field that arises at plasmon resonance. Upon colliding at the interface, the excited $\mathrm{Ag} \mathrm{CB}$ electrons (which may be hot electrons of the plasmon excitation) are temporarily transferred to the $\mathrm{Al}_{2} \mathrm{O}_{3} \mathrm{CB}$ and back again. In this qualitative picture, the energy difference between $\mathrm{CB}$ of $\mathrm{Ag}$ and alumina layers is levelled by the Schottky potential. In addition, interfaces are playing a complex role in a heat transfer process. The increment of heat in alumina with transferred electrons can be dissipated by their return to the Ag layer. This process is controlled by interface scattering; returned electrons will be involved in plasmon excitation, etc. As we have shown in Figure 10b,c, the spatial distribution of plasmon excitations is very complex near boundaries and in nanosized volumes. On one hand, the contact of $\mathrm{Ag}$ with alumina promotes heat transfer by the electronic mechanism, and on the other hand, confinement and amplification of inelastic scattering by the interface greatly weakens this heat flux. In planar nanostructures materials, its heat conductivity will decrease until only electron 
tunnelling is permitted. Tunnelling of electrons between Ag layers will suddenly and radically increase the thermal conductivity of the material when the thickness of $\mathrm{Al}_{2} \mathrm{O}_{3}$ reaches several nm or less [49].

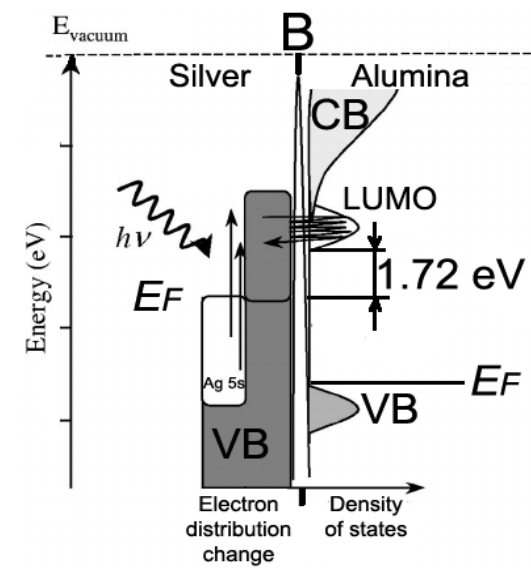

Figure 11. Scheme of physical conditions for plasmon-polariton excitation at the interface of $\mathrm{Ag}$ and $\mathrm{Al}_{2} \mathrm{O}_{3}$ nanolayers. $\mathrm{B}$-interface; $\mathrm{VB}$ - valence band; $\mathrm{CV}$ - conducting band; $\mathrm{EF}$-Fermi level; LUMO_lowest unoccupied molecular orbital.

\section{Conclusions}

The anomalous reduction of thermal conductivity in nanolaminate multilayer $\mathrm{Ag} / \mathrm{Al}_{2} \mathrm{O}_{3}$ coating in comparison with bulk components is explained by the influence of confinement on plasmons and phonons' propagation. This is based on a considerable decline of heat conductivity in the set of nanoscaled $\mathrm{Ag} / \mathrm{Al}_{2} \mathrm{O}_{3}$ multilayered coatings with individual layer thickness decreasing from 170 to $1 \mathrm{~nm}$. Nanostructuring influences the electronic structures of metal and insulator layers. Binding energies of electrons on inner levels in $\mathrm{Ag}$ atoms increase, and this localization of electrons reduces the metal character of interatomic bonds when the layer thickness is decreased. The band gap in alumina evolves oppositely in points $\Gamma$ and $X$ of zone structure at layer thinning: in $170 \mathrm{~nm}$ layer, these values correspond to 6.6 and $8.45 \mathrm{eV}$; and they are changed to 6.9 and $8.15 \mathrm{eV}$ in $24 \mathrm{~nm}$ layers and to 8.20 and $7.5 \mathrm{eV}$ in $10 \mathrm{~nm}$ layers, accordingly. The size effect of nanostructuring is expressed by the increasing of the effective band gap due to its smoothing.

The contribution of phonon propagation to the heat transfer mechanism in $\mathrm{Ag} / \mathrm{Al}_{2} \mathrm{O}_{3}$ nanolayer composites has several features. The process obeys the classical picture when thinning of layers below the phonon MFP in the nanometer range reduces the intensity of energy transport by phonons. Anisotropy of acoustic phonons' propagation rises when reducing the individual layers in the multilayer coating. Furthermore, the probability of phonon transmittance across $\mathrm{Ag} / \mathrm{Al}_{2} \mathrm{O}_{3}$ interfaces significantly decreases with layer thinning. On the contrary, the probability of opposite phonon transportation in direction $\mathrm{Al}_{2} \mathrm{O}_{3} / \mathrm{Ag}$ increases. Despite such a complex physical picture, the contribution of the interface phonon heat transfer to the overall balance is very small, but it increases with decreasing layer thickness.

Plasmon resonance propagates in the volume of the metal in a symmetric or antisymmetric manner depending on the excitation energy and thickness of silver layers surrounded by alumina. This non-uniform resonance structure is a consequence of the confinement of electromagnetic density of states in a thin planar nanocomposite. The plasmon-polariton interaction propagates $2.0-5.0 \mathrm{~nm}$ into $\mathrm{Al}_{2} \mathrm{O}_{3}$, and depends proportionally on the intensity of plasmon excitation in $\mathrm{Ag}$. Plasmon-polariton propagation is the main electronic mechanism of heat transfer. Electronic DOS at $E_{\mathrm{F}}$ in alumina is too low to rise above the wide band gap by internal interband transitions. Polariton resonance results in injection of $\mathrm{Ag} 5 \mathrm{~s}$ electrons into $\mathrm{CB}$ of $\mathrm{Al}_{2} \mathrm{O}_{3}$ by energy pumping and the boundary electromagnetic field generated by plasmon resonance. The layer thinning in the investigated multilayer coatings 
complicates plasmons and phonons' propagation in layers, and at interfaces and it is a key reason for the sharp heat conductivity lessening.

Author Contributions: Conceptualization, A.K., D.W., and J.L.E.; investigation, D.W., A.K., V.V., and R.G.; formal analysis, V.V.; visualization, D.W., A.K., and V.V.; writing-original draft preparation, D.W. and A.K.; writing-review and editing, D.W., A.K., V.V., R.G., and J.L.E. All authors have read and agreed to the published version of the manuscript.

Funding: The research was carried out with partial financial support of RSF, project number 14-12-00170 (in part, for the fabrication and characterization of $\mathrm{Al}_{2} \mathrm{O}_{3} / \mathrm{Ag}$ coatings) and RFBR project number 16-08-00527 (modelling of plasmons and phonons propagation).

Conflicts of Interest: The authors declare no conflict of interest.

\section{References}

1. Mosquera, A.A.; Horvat, D.; Rashkovskiy, A.; Kovalev, A.; Miska, P.; Wainstein, D.; Allbella, J.; Endrino, J.L. Exciton and core-level electron confinement effects in transparent ZnO thin films. Sci. Rep. 2013, 3, 1714. [CrossRef]

2. Gao, Y.; Zhao, X.; Yin, P.; Gao, F. Size-Dependent Raman Shifts for nanocrystals. Sci. Rep. 2016, 6, 20539. [CrossRef] [PubMed]

3. Yao, K.; Liu, Y. Plasmonic metamaterials. Nanotech. Rev. 2014, 3, 177-210. [CrossRef]

4. Sun, J.; Shalaev, M.I.; Litchinitser, N.M. Experimental demonstration of a non-resonant hyperlens in the visible spectral range. Nat. Commun. 2015, 6, 7201. [CrossRef]

5. Callebaut, H.; Kumar, S.; Williams, B.S.; Hua, Q.; Reno, J.L. Importance of electron-impurity scattering for electron transport in terahertz quantum-cascade lasers. Appl. Phys. Lett. 2004, 84, 645-647. [CrossRef]

6. Shen, X.; Cui, T. Planar plasmonic metamaterial on a thin film with nearly zero thickness. Appl. Phys. Lett. 2013, 102, 211909. [CrossRef]

7. Tian, Z.; Esfarjani, K.; Shiomi, J.; Henry, A.S.; Chen, G. On the importance of optical phonons to thermal conductivity in nanostructures. Appl. Phys. Lett. 2011, 99, 053122. [CrossRef]

8. Kargar, F.; Ramirez, S.; Debnath, B.; Malekpour, H.; Lake, R.; Balandin, A.A. Acoustic Phonon Spectrum and Thermal transport in Nanoporous Alumina Arrays. Appl. Phys. Lett. 2015, 107, 171904. [CrossRef]

9. Toberer, E.S.; Baranowski, L.L.; Dames, C. Advances in thermal conductivity. Annu. Rev. Mater. Res. 2012, 42, 179-209. [CrossRef]

10. Cahill, D.G. Nanoscale thermal transport. Appl. Phys. Rev. 2014, 1, 011305. [CrossRef]

11. Balandin, A.A.; Denis, L.N. Phononics in low-dimensional materials. Mater. Today 2012, 15, $266-275$. [CrossRef]

12. Bell, L.E. Cooling, heating, generating power, and recovering waste heat with thermoelectric systems. Science 2015, 321, 1457-1461. [CrossRef] [PubMed]

13. Biswas, K. High-performance bulk thermoelectrics with all-scale hierarchical structures. Nature 2012, 489, 414-418. [CrossRef] [PubMed]

14. Oraizi, H.; Abdolali, A. Design and optimization of planar multilayer antireflection metamaterial coatings at KU band under circularly polarized oblique plane wave incidence. Prog. Electromagn. Res. C 2008, 3, 1-18. [CrossRef]

15. Chen, J.; Zhang, G.; Li, B. Impacts of Atomistic Coating on Thermal Conductivity of Germanium Nanowires. Nano Lett. 2012, 12, 2826-2832. [CrossRef]

16. Moldovan, M. Phonon wave interference and thermal bandgap materials. Nat. Mater. 2015, 14, 667-674. [CrossRef]

17. Chou, Y.-H.; Wu, Y.; Hong, K.-B.; Chou, B.-T.; Shih, J.-H.; Chung, Y.-C.; Chen, P.-Y.; Lin, T.-R.; Lin, C.-C.; Lin, S.-D.; et al. High-operation-temperature plasmonic nanolasers on single-crystalline aluminum. Nano Lett. 2016, 16, 3179-3186. [CrossRef]

18. Ordonez-Miranda, J.; Tranchant, L.; Chalopin, Y.; Antoni, T.; Volz, S. Thermal conductivity of nano-layered systems due to surface phonon-polaritons. Journ. Appl. Phys. 2015, 115, 054311. [CrossRef]

19. Chen, D.Z.A.; Narayanaswamy, A.; Chen, G. Enhancement of in-plane thermal conductivity of thin films via surface phonon-polaritons. Proceedings of IMECE2005 ASME International Mechanical Engineering Congress and Exposition, Orlando, FL, USA, 5-11 November, 2005; pp. 841-846. 
20. Kovalev, A.I.; Wainstein, D.L.; Rashkovskiy, A.Y.; Osherov, A.; Golan, Y. Size shift of XPS lines observed from PbS nanocrystals. Surf. Interf. Anal. 2010, 42, 850-854. [CrossRef]

21. Kovalev, A.I.; Wainshtein, D.L.; Rashkovskiy, A.Y.; Golan, Y.; Osherov, A.; Ashkenazy, N. Studying of Quantum-Size Effects Origination in Semiconducting Lead Sulfide Nanocrystals. Protect. Met. Phys. Chem. Surf. 2010, 46, 633-638. [CrossRef]

22. Kovalev, A.; Wainstein, D.; Rashkovskiy, A.; Golan, Y.; Osherov, A.; Ashkenasi, N. Complex investigation of electronic structure transformations in Lead Sulfide nanoparticles using a set of electron spectroscopy techniques. Vacuum 2012, 86, 638-642. [CrossRef]

23. Wainstein, D.L.; Kovalev, A.I. Regularities of electronic structure transformations in nanomaterials with decreasing their characteristic size. J. Phys. Conf. Ser. 2017, 857, 012055. [CrossRef]

24. Kovalev, A.I.; Wainstein, D.L.; Rashkovskiy, A.Y.; Gago, R.; Soldera, F.; Endrino, J.L.; Fox-Rabinovich, G.S. Interface-Induced Plasmon Nonhomogeneity in Nanostructured Metal-Dielectric Planar Metamaterial. J. Nanomat. 2015, 2015, 231-240. [CrossRef]

25. Kovalev, A.I.; Rashkovskiy, A.Y.; Wainstein, D.L.; Gago, R.; Soldera, F.; Endrino, J.L. Influence of electronic structure, plasmon-phonon and plasmon-polariton excitations on anomalously low heat conductivity in TiAlN/Ag nanoscale multilayer coatings. Curr. Appl. Phys. 2016, 16, 459-468. [CrossRef]

26. Kovalev, A.I.; Wainstein, D.L.; Rashkovskiy, A.Y.; Gago, R.; Soldera, F.; Endrino, J.L. The confinement of phonon propagation in TiAlN/Ag multilayer coatings with anomalously low heat conductivity. Appl. Phys. Lett. 2016, 108, 223106. [CrossRef]

27. Gago, R.; Soldera, F.; Huebner, R.; Lehmann, J.; Munnik, F.; Vázquez, L.; Redondo-Cubero, A.; Endrino, J.L. $\mathrm{X}$-ray absorption near-edge structure of hexagonal ternary phases in sputter-deposited TiAlN films. J. Alloys Compd. 2013, 561, 87-94. [CrossRef]

28. Mayer, M. SIMNRA User's Guide 6.05; Max-Planck-Institut für Plasmaphysik: Garching, Germany, 2009.

29. Cahill, D.G. Analysis of heat flow in layered structures for time-domain thermoreflectance. Rev. Sci. Instrum. 2004, 75, 5119-5122. [CrossRef]

30. Josell, D.; Bonevich, J.E.; Nguyen, T.M.; Johnson, R.N. Heat transfer through nanoscale multilayered thermal barrier coatings at elevated temperatures. Surf. Coat. Tech. 2015, 275, 75. [CrossRef]

31. Jain, A.; McGaughey, A.J.H. Thermal transport by phonons and electrons in aluminum, silver, and gold from first principles, Supplementary Information. Phys. Rev. B 2016, 93, 081206. [CrossRef]

32. Majumdar, A. Microscale heat conduction in dielectric thin films. ASME HTD 1991, 184, 34-41. [CrossRef]

33. Jennifer Su, Y.; Wang, H.; Porter, W.D.; De Arellano Lopez, A.R.; Faber, K.T. Thermal conductivity and phase evolution of plasma-sprayed multilayer coatings. J. Mater. Sci. 2001, 36, 3511-3518.

34. Luo, Z.; Liu, H.; Feng, Y.; Ye, P.; Chen, Y.; Xu, X. In-plane thermal conductivity of ultra-thin $\mathrm{Al}_{2} \mathrm{O}_{3}$ films measured by Micro-Raman. In Proceedings of the ASME 2013 Heat Transfer Summer Conference HT2013, Minneapolis, MN, USA, 14-19 July 2013; pp. HT2013-17170.

35. Stark, I.; Stordeur, M.; Syrowatka, F. Thermal conductivity of thin amorphous alumina films. Thin Solid Films 1993, 226, 185-190. [CrossRef]

36. Cappella, A.; Battaglia, J.-L.; Schick, V.; Kusiak, A.; Lamperti, A.; Wiemer, C.; Hay, B. High temperature thermal conductivity of amorphous $\mathrm{Al}_{2} \mathrm{O}_{3}$ thin films grown by low temperature ALD. Adv. Eng. Mater. 2013, 15, 9999. [CrossRef]

37. Heino, P.; Ristolainen, E. Thermal conduction at the nanoscale in some metals by MD. Microelectron. J. 2003, 34, 773-777. [CrossRef]

38. Ryu, S.; Juhng, W.; Kim, Y. Effect of microstructure on thermal conductivity of Cu, Ag thin films. J. Nanosci. Nanotechnol. 2010, 10, 3406-3411. [CrossRef]

39. Cocemasov, A.I.; Nika, D.L.; Fomin, V.M.; Grimm, D.; Schmidt, O.G. Phonon-engineered thermal transport in Si wires with constant and periodically modulated cross-sections: A crossover between nano- and microscale regimes. Appl. Phys. Lett. 2015, 107, 011904. [CrossRef]

40. Perevalov, T.V.; Shaposhnikov, A.V.; Gritsenko, V.A.; Wong, H.; Han, J.H.; Kim, C.W. Electronic structure of $\alpha-\mathrm{Al}_{2} \mathrm{O}_{3}$ : Ab initio simulations and comparison with experiment. JETP Lett. 2007, 85, 165-168. [CrossRef]

41. Liu, D.M.; Tuan, W.H. Microstructure and thermal condu $\mathrm{u}_{\mathrm{c}} \mathrm{t}_{\mathrm{i}}$ on properties $\mathrm{Al}_{2} \mathrm{O}_{3}-\mathrm{Ag}$ composites. Acta mater. 1996, 44, 813-818. [CrossRef]

42. Mousavi, S.J.; Abolhassani, M.R.; Hosseini, S.M.; Sebt, S.A. Comparison of electronic and optical properties of the $\alpha$ and $\mathrm{k}$ phases of alumina using density functional theory. Chin. J. Phys. 2009, 47, 862-873. 
43. Yazdanmehr, M.; Asadabadi, S.J.; Nourmohammadi, A.; Ghasemzadeh, M.; Rezvanian, M. Electronic structure and bandgap of $\gamma-\mathrm{Al}_{2} \mathrm{O}_{3}$ compound using mBJ exchange potential. Nanoscale Res. Lett. 2012, 7, 488. [CrossRef]

44. Bialas, H.; Stolz, H.J. Lattice dynamics of sapphire (corundum). Part I: Phonon dispersion by inelastic neutron scattering. Z. Physik B 1975, 21, 319-324. [CrossRef]

45. Zhu, K.-R.; Zhang, M.-S.; Chen, Q.; Yin, Z. Size and phonon-confinement effects on low-frequency Raman mode of anatase TiO2 nanocrystal. Phys. Lett. A 2005, 340, 220-227. [CrossRef]

46. Henrique, T.M.C.; Baltar, M.; Drozdowicz-Tomsia, K.; Goldys, E.M. Propagating surface plasmons and dispersion relations for nanoscale multilayer metallic-dielectric films. In Plasmonics-Principles and Applications; Kim, Y., Ed.; InTech: Moscow, Ruassia, 2012.

47. Nelayah, J.; Kociak, M.; Stéphan, O.; Javier García de Abajo, F.; Tencé, M.; Henrard, L.; Taverna, D.; Pastoriza-Santos, I.; Liz-Marzán, L.M.; Colliex, C. Mapping surface plasmons on a single metallic nanoparticle. Nat. Phys. 2007, 3, 348-353. [CrossRef]

48. Singh, K.; Hammond, S.N.A. Current-voltage characteristics and photoresponse of metal-metal devices. Turk. J. Phys. 1998, 22, 315.

49. Zhang, P. Scaling for quantum tunneling current in nano- and subnano-scale plasmonic junctions. Sci. Rep. 2015, 5, 9826. [CrossRef]

(C) 2020 by the authors. Licensee MDPI, Basel, Switzerland. This article is an open access article distributed under the terms and conditions of the Creative Commons Attribution (CC BY) license (http://creativecommons.org/licenses/by/4.0/). 